\title{
Microwave-assisted cyclizations promoted by polyphosphoric acid esters: a general method for 1-aryl-2-iminoazacycloalkanes
}

\author{
Jimena E. Díaz, María C. Mollo and Liliana R. Orelli*
}

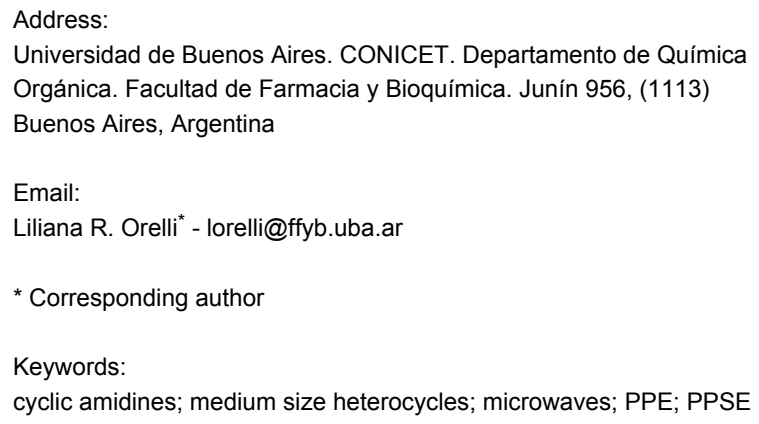

\author{
Beilstein J. Org. Chem. 2016, 12, 2026-2031. \\ doi:10.3762/bjoc. 12.190 \\ Received: 27 May 2016 \\ Accepted: 30 August 2016 \\ Published: 14 September 2016 \\ Associate Editor: B. Stoltz \\ () 2016 Díaz et al.; licensee Beilstein-Institut. \\ License and terms: see end of document.
}

\begin{abstract}
The first general procedure for the synthesis of 5 to 7-membered 1-aryl-2-iminoazacycloalkanes is presented, by microwaveassisted ring closure of $\omega$-arylaminonitriles promoted by polyphosphoric acid (PPA) esters. 1-Aryl-2-iminopyrrolidines were easily prepared from the acyclic precursors employing a chloroformic solution of ethyl polyphosphate (PPE). The use of trimethylsilyl polyphosphate (PPSE) in solvent-free conditions allowed for the synthesis of 1-aryl-2-iminopiperidines and hitherto unreported 1-aryl-2-iminoazepanes. The cyclization reaction involves good to high yields and short reaction times, and represents a novel application of PPA esters in heterocyclic synthesis.
\end{abstract}

\section{Introduction}

The synthesis of new nitrogen heterocycles has a great interest in medicinal chemistry since they are part of many drugs and represent structures with a wide therapeutic potential $[1,2]$. 2-Iminoazacycloalkanes are cyclic amidines where the formally $\mathrm{sp}^{2}$ nitrogen is exocyclic. These compounds have been described as selective inhibitors of the inducible form of human nitric oxide synthase (iNOS), which catalyzes the reaction to form nitric oxide via the oxidation of L-arginine to L-citrulline [3-6]. Some urea and thiourea derivatives have been studied as CNS agents [7] and anthelmintic drugs [8]. This heterocyclic core therefore represents the foundation for potential bioactive agents.
A few methods have been described for the synthesis of 2-iminoazacycloalkanes from acyclic precursors. One strategy involves the cyclization of $\omega$-aminonitriles, which requires the presence of protic acids $[9,10]$. It has also been described the reaction between halonitriles and primary or secondary amines, where the $\omega$-aminonitrile is formed in situ [8,11-14]. This heterocyclic core has also been prepared by cyclization of azidonitriles [15]. These methods usually require drastic reaction conditions and are generally limited to 2-iminopyrrolidines and/or to $\mathrm{N}$-unsubstituted or $\mathrm{N}$-alkyl derivatives. Other methods involve cyclic precursors such as lactams $[4,6,7,16]$, thiolactams $[3,6]$ or 2-aminopyridines [6]. 
Literature examples on the corresponding $N$-unsubstituted or $\mathrm{N}$-alkyl seven membered heterocycles, i.e. 2-iminoazepanes $[3,7,9,10]$, are very scarce, and no general method has been described for their synthesis. To our knowledge, $\mathrm{N}$-aryl derivatives have not been hitherto reported. The lack of synthetic methods can be attributed to the intrinsic difficulty of cyclizations leading to seven membered heterocycles. This is a consequence of the high activation energies involved in these reactions, which are also hindered by entropic factors [17-22]. In addition to this, due to the considerably lower nucleophilicity of the arylamino group, procedures for $\mathrm{N}$-alkyl or $\mathrm{N}$-unsubstituted 2-iminoazacycloalkanes are usually not suitable for the synthesis of $N$-aryl derivatives.

Ethyl polyphosphate (PPE) and trimethylsilyl polyphosphate (PPSE) are mild irreversible dehydrating agents of the Lewis acid type. They have been widely used for several synthetically useful transformations like dehydration of amides leading to nitriles $[23,24]$ or the Beckmann rearrangement $[23,25]$. They have also been employed in the synthesis of heterocycles such as benzimidazoles, benzothiazoles, benzoxazoles [23,26-28], indoles and 3,4-dihydroisoquinolines [23,29]. Microwaveassisted organic reactions proceed in general faster, with higher yields and more efficiently than those performed under conventional heating [30-38]. This technology has found interesting applications in heterocyclic synthesis, specially in cyclocondensation reactions $[39,40]$. In particular, it has enabled the synthesis of the more challenging medium size (7-9 membered) heterocycles by overcoming limitations such as low reactivity and yields, harsh reaction conditions and side reactions typical of conventional heating [17].

In previous work, we reported the microwave-assisted synthesis of 5 to 8 membered cyclic amidines by cyclodehydration of $N$-aryl- $N$ '-acyl-1, $n$-alkanediamines $(n=2-5)$ promoted by polyphosphoric acid (PPA) esters PPE and PPSE (Scheme 1, reaction 1) [41-43]. In this case, PPA esters activate the amidic oxygen and, at the same time, react chemically with water.

We hypothesized that applying similar reaction conditions to $\omega$-arylaminonitriles could lead to 1-aryl-2-imino-1-azacycloalkanes (Scheme 1, reaction 2), providing a novel synthetic method for these heterocycles. In this case no dehydration would be involved and PPA esters, due to their Lewis acid nature, would solely increase the electrophilicity of the cyano group towards an intramolecular nucleophilic attack. This approach would avoid the use of strong protic acids, which may be disadvantageous for sensitive substrates.

\section{Results and Discussion}

The $\omega$-arylaminonitrile precursors were obtained by reaction of the corresponding $\omega$-halonitrile and arylamines, as previously reported by our group [44].

We examined first the cyclization of 4-(p-tolylamino)butyronitrile (1a) with PPE under microwave irradiation in a closed vessel reactor. The reaction was completed after 5 minutes at $100{ }^{\circ} \mathrm{C}$ and 1-( $p$-tolyl)-2-iminopyrrolidine (2a) was obtained in $86 \%$ yield (Table 1 , entry 1 ). No reaction occurred in the absence of PPE, while the use of classical Lewis acids $\left(\mathrm{ZnCl}_{2}\right.$, $\mathrm{AlCl}_{3}, \mathrm{BF}_{3}$ ) as cyclization agents led to very low yields of the desired product 2a (Table 1, entries 2-5).

Employing the optimized experimental conditions, 1-aryl-2iminopyrrolidines $\mathbf{2 b}-\mathbf{h}$ were prepared in high yields (Table 1, entries 6-12).

In order to extend the scope of the method, we investigated next the synthesis of 1-aryl-2-iminopiperidines 4 . The conversion of 5-( $p$-tolylamino)valeronitrile (3a) to 1-( $p$-tolyl)-2-iminopiperidine (4a) was chosen for the optimization of the reaction conditions (Table 2). In the conditions used for the lower homo-
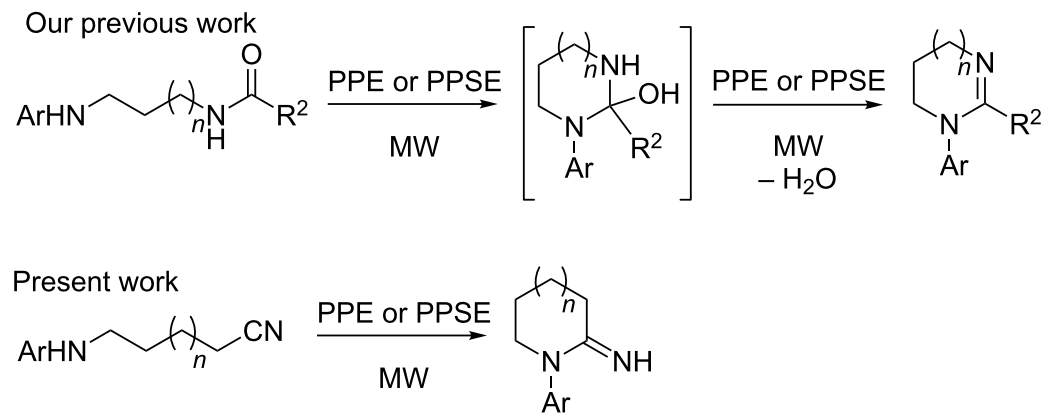
Table 1: Synthesis of 1-aryl-2-iminopyrrolidines $2 \mathrm{a}-\mathrm{h}$.

\begin{tabular}{|c|c|c|c|}
\hline & 1 & $100^{\circ} \mathrm{C}, 5 \mathrm{~min}$ & Ar 2 \\
\hline Entry & Compound 2 & $\mathrm{Ar}$ & Yield (\%) \\
\hline 1 & $\mathbf{a}$ & $4-\mathrm{CH}_{3} \mathrm{C}_{6} \mathrm{H}_{4}$ & 86 \\
\hline $2^{a}$ & $\mathbf{a}$ & $4-\mathrm{CH}_{3} \mathrm{C}_{6} \mathrm{H}_{4}$ & 0 \\
\hline $3^{b}$ & $\mathbf{a}$ & $4-\mathrm{CH}_{3} \mathrm{C}_{6} \mathrm{H}_{4}$ & traces \\
\hline $4^{c}$ & a & $4-\mathrm{CH}_{3} \mathrm{C}_{6} \mathrm{H}_{4}$ & 11 \\
\hline $5^{d}$ & a & $4-\mathrm{CH}_{3} \mathrm{C}_{6} \mathrm{H}_{4}$ & 17 \\
\hline 6 & b & $\mathrm{C}_{6} \mathrm{H}_{5}$ & 85 \\
\hline 7 & c & $4-\mathrm{FC}_{6} \mathrm{H}_{4}$ & 74 \\
\hline 8 & d & 4- $\mathrm{ClC}_{6} \mathrm{H}_{4}$ & 82 \\
\hline 9 & e & $4-\mathrm{BrC}_{6} \mathrm{H}_{4}$ & 77 \\
\hline 10 & $f$ & $2-\mathrm{CH}_{3} \mathrm{C}_{6} \mathrm{H}_{4}$ & 77 \\
\hline 11 & g & $2-\mathrm{ClC}_{6} \mathrm{H}_{4}$ & 80 \\
\hline $12^{\mathrm{e}}$ & h & $2-\mathrm{OCH}_{3} \mathrm{C}_{6} \mathrm{H}_{4}$ & 85 \\
\hline
\end{tabular}

aThe reaction was run in the absence of reagents (background reaction). ${ }^{b}$ The reaction was run using stoichiometric amount of $\mathrm{ZnCl}_{2}$. ${ }^{c}$ The reaction was run using stoichiometric amount of $\mathrm{AlCl}_{3}$. ${ }^{\mathrm{d}} \mathrm{The}$ reaction was run using stoichiometric amount of $\mathrm{BF}_{3}$. ${ }^{\mathrm{e}} \mathrm{The}$ reaction time was 20 minutes.

logues $2\left(\mathrm{PPE} / \mathrm{CHCl}_{3}, 100{ }^{\circ} \mathrm{C}\right)$, no reaction occurred after 5 minutes (Table 2, entry 1). Increasing the reaction time and/or temperature resulted in higher conversion, but the $N$-ethyl derivative 5a was obtained along with the desired product (Table 2, entries 2 and 3). This side reaction was quite unexpected, although some examples in the literature show that PPE can act as an ethylating agent under certain conditions $[45,46]$. Using a dichloromethane solution of PPSE, compound $\mathbf{4 a}$ was obtained exclusively (Table 2, entries 4-6), while working under solvent-free conditions further improved the yield (Table 2, entry 7).

Employing the optimized reaction conditions (neat PPSE, $30 \mathrm{~min}$ at $150{ }^{\circ} \mathrm{C}$ ), 1-aryl-2-iminopiperidines 4 were synthesized in high yields (Table 3 ). Compound $\mathbf{4 g}$ required a higher reaction temperature $\left(200^{\circ} \mathrm{C}\right)$ due to steric hindrance of the $N$-aryl moiety.

The encouraging results obtained up to this point, together with the absence of methods for the synthesis of the higher homologues 1-aryl-2-iminoazepanes 7 prompted us to attempt the microwave-assisted cyclization of 6-arylaminohexanenitriles 6 promoted by PPA esters. We examined first the cyclization of compound 6a under different reaction conditions (Table 4). No conversion was observed using $\mathrm{PPE} / \mathrm{CHCl}_{3}$ at $100{ }^{\circ} \mathrm{C}$ for 5 minutes (Table 4, entry 1). When longer reaction times and/or higher temperatures were used, 1-( $p$-tolyl)-2-ethyliminoazepane (8a) was obtained as the only product (Table 4 , entries 2 and 3). The use of PPSE in DCM solution afforded traces of the desired product 7a (Table 4, entry 4). Performing the reaction under solvent-free conditions at $150{ }^{\circ} \mathrm{C}$, compound $7 \mathbf{a}$ was obtained in modest yield (Table 4, entry 5). Significantly better results were achieved by increasing the temperature to $200{ }^{\circ} \mathrm{C}$ (Table 4 , entry 6 ).

Employing the optimized reaction conditions, we synthesized some novel 1-aryl-2-iminoazepanes 7 in good to high yields (Table 5). A clear steric effect on the reactivity was observed in the case of compound $\mathbf{7 g}$ (Table 5 , entry 7), which resulted in low conversion of the substrate.

Table 2: Reaction conditions screening for 1-( $p$-tolyl)-2-iminopiperidine (4a).

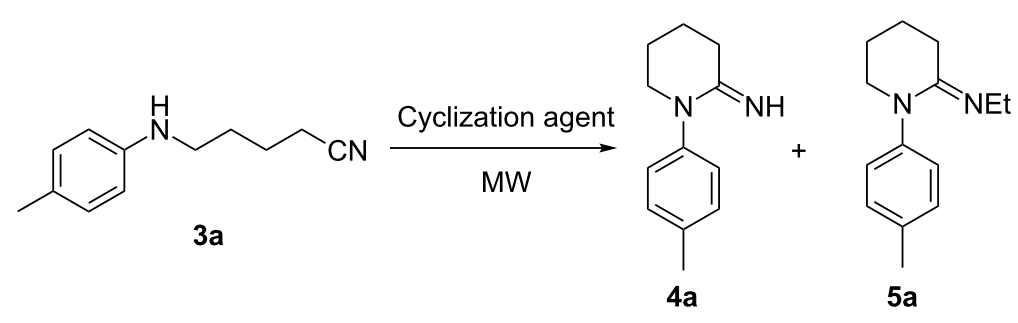

\begin{tabular}{|c|c|c|c|c|c|c|}
\hline Entry & Cyclization agent & Solvent & Temp. $\left({ }^{\circ} \mathrm{C}\right)$ & Time (min.) & $4 a(\%)$ & $5 a(\%)$ \\
\hline 1 & PPE & $\mathrm{CHCl}_{3}$ & 100 & 5 & 0 & 0 \\
\hline 2 & PPE & $\mathrm{CHCl}_{3}$ & 100 & 30 & traces & traces \\
\hline 3 & PPE & $\mathrm{CHCl}_{3}$ & 150 & 30 & 32 & 32 \\
\hline 4 & PPSE & $\mathrm{CH}_{2} \mathrm{Cl}_{2}$ & 130 & 15 & 34 & 0 \\
\hline 5 & PPSE & $\mathrm{CH}_{2} \mathrm{Cl}_{2}$ & 150 & 15 & 62 & 0 \\
\hline 6 & PPSE & $\mathrm{CH}_{2} \mathrm{Cl}_{2}$ & 150 & 30 & 71 & 0 \\
\hline 7 & PPSE & none & 150 & 30 & 74 & 0 \\
\hline
\end{tabular}


Table 3: Synthesis of 1-aryl-2-iminopiperidines 4.

\begin{tabular}{|c|c|c|c|}
\hline & 3 & $150^{\circ} \mathrm{C}, 30 \mathrm{~min}$ & \\
\hline Entry & Compound 4 & $\mathrm{Ar}$ & Yield (\%) \\
\hline 1 & $\mathbf{a}$ & 4- $\mathrm{CH}_{3} \mathrm{C}_{6} \mathrm{H}_{4}$ & 74 \\
\hline 2 & b & $\mathrm{C}_{6} \mathrm{H}_{5}$ & 79 \\
\hline 3 & C & $4-\mathrm{ClC}_{6} \mathrm{H}_{4}$ & 72 \\
\hline $4^{a}$ & d & $2-\mathrm{CH}_{3} \mathrm{C}_{6} \mathrm{H}_{4}$ & 74 \\
\hline 5 & e & $2-\mathrm{FC}_{6} \mathrm{H}_{4}$ & 82 \\
\hline $6^{a}$ & f & $2-\mathrm{ClC}_{6} \mathrm{H}_{4}$ & 73 \\
\hline $7^{\mathrm{b}}$ & g & $2,6-\left(\mathrm{CH}_{3}\right)_{2} \mathrm{C}_{6} \mathrm{H}_{4}$ & 86 \\
\hline
\end{tabular}

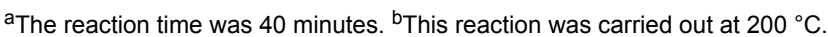

Table 4: Reaction conditions screening for 1-( $p$-tolyl)-2-iminoazepane (7a).<smiles>Cc1ccc(NCCCCCC#N)cc1</smiles>

$6 a$<smiles>CCC1=NC(=O)CCCCCN1c1ccc(Br)cc1</smiles>

$8 a$

\begin{tabular}{ccccccc}
\hline Entry & Cyclization agent & Solvent & Temp. $\left({ }^{\circ} \mathrm{C}\right)$ & Time $(\mathrm{min})$ & $\mathbf{7 a}(\%)$ & $\mathbf{8 a}(\%)$ \\
\hline 1 & PPE & $\mathrm{CHCl}_{3}$ & 100 & 5 & 0 & 0 \\
2 & PPE & $\mathrm{CHCl}_{3}$ & 100 & 30 & 0 & 10 \\
3 & PPE & $\mathrm{CHCl}_{3}$ & 150 & 30 & 0 & 77 \\
4 & PPSE & $\mathrm{CH}_{2} \mathrm{Cl}_{2}$ & 150 & 30 & traces & 0 \\
5 & PPSE & none & 150 & 30 & 45 & 0 \\
6 & PPSE & none & 200 & 30 & 73 & 0
\end{tabular}

Table 5: Synthesis of 1-aryl-2-iminoazepanes 7.

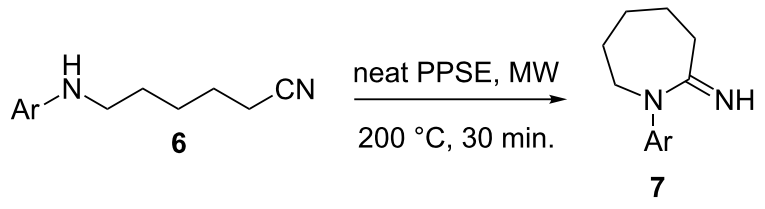

\begin{tabular}{cccc}
\hline Entry & Compound 7 & Ar & Yield (\%) \\
\hline 1 & a & 4- $\mathrm{CH}_{3} \mathrm{C}_{6} \mathrm{H}_{4}$ & 73 \\
2 & b & $\mathrm{C}_{6} \mathrm{H}_{5}$ & 62 \\
3 & c & $4-\mathrm{FC}_{6} \mathrm{H}_{4}$ & 75 \\
4 & d & $4-\mathrm{BrC}_{6} \mathrm{H}_{4}$ & 55 \\
5 & e & $2-\mathrm{CH}_{3} \mathrm{C}_{6} \mathrm{H}_{4}$ & 68 \\
7 & f & $2-\mathrm{FC}_{6} \mathrm{H}_{4}$ & 28
\end{tabular}




\section{Conclusion}

In summary, we have developed a straightforward and efficient protocol for the microwave-assisted synthesis of 1-aryl-2-iminoazacycloalkanes, by ring closure of $\omega$-arylaminonitriles promoted by PPA esters. This reaction constitutes a novel application of such reagents in heterocyclic synthesis. The procedure involves easily available starting materials and requires remarkably short reaction times. It affords the desired compounds in good to high yields and avoids the use of protic acids. Noteworthly, classical Lewis acids failed to efficiently promote this transformation. To our knowledge, this is the first method which allows the synthesis of the hitherto unreported $N$-aryl 7-membered heterocycles.

\section{Supporting Information}

\section{Supporting Information File 1}

Experimental procedures, characterization of new compounds and copies of ${ }^{1} \mathrm{H}$ and ${ }^{13} \mathrm{C}$ NMR spectra. [http://www.beilstein-journals.org/bjoc/content/ supplementary/1860-5397-12-190-S1.pdf]

\section{Acknowledgements}

This work was supported by the University of Buenos Aires (20020130100466). We are grateful to Prof. A. Moglioni for providing access to the microwave reactor.

\section{References}

1. Baumann, M.; Baxendale, I. R.; Ley, S. V.; Nikbin, N. Beilstein J. Org. Chem. 2011, 7, 442-495. doi:10.3762/bjoc.7.57

2. Baumann, M.; Baxendale, I. R. Beilstein J. Org. Chem. 2013, 9 , 2265-2319. doi:10.3762/bjoc.9.265

3. Moore, W. M.; Webber, R. K.; Fok, K. F.; Jerome, G. M.; Connor, J. R.; Manning, P. T.; Wyatt, P. S.; Misko, T. P.; Tjoeng, F. S.; Currie, M. G. J. Med. Chem. 1996, 39, 669-672. doi:10.1021/jm950766n

4. Hagen, T. J.; Bergmanis, A. A.; Kramer, S. W.; Fok, K. F.; Schmelzer, A. E.; Pitzele, B. S.; Swenton, L.; Jerome, G. M.; Kornmeier, C. M.; Moore, W. M.; Branson, L. F.; Connor, J. R.; Manning, P. T.; Currie, M. G.; Hallinan, E. A. J. Med. Chem. 1998, 41, 3675-3683. doi:10.1021/jm970840x

5. Shankaran, K.; Donnelly, K. L.; Shah, S. K.; Guthikonda, R. N.; MacCoss, M.; Humes, J. L.; Pacholok, S. G.; Grant, S. K.; Kelly, T. M.; Wong, K. K. Bioorg. Med. Chem. Lett. 2004, 14, 4539-4544. doi:10.1016/j.bmcl.2004.06.033

6. Webber, R. K.; Metz, S.; Moore, W. M.; Connor, J. R.; Currie, M. G.; Fok, K. F.; Haggen, T. J.; Hansen, D. W.; Jerome, G. M.; Manning, P. T.; Pitzele, B. S.; Toth, M. V.; Trivedi, M.; Zupec, M. E.; Tjoeng, F. S. J. Med. Chem. 1998, 41, 96-101. doi:10.1021/jm9705059

7. Rasmussen, C. R.; Gardocki, J. F.; Plampin, J. N.; Twardzik, B. L.; Reynolds, B. E.; Molinari, A. J.; Schwartz, N.; Bennetts, W. W.; Price, B. E.; Marakowski, J. J. Med. Chem. 1978, 21, 1044-1054. doi:10.1021/jm00208a008
8. Diana, G. D. 1-Phenyl-azacarbocyclic-2-imines. U.S. Patent 3717648, Feb 20, 1973.

9. Blicke, F. F.; Zambito, A. J.; Stenseth, R. E. J. Org. Chem. 1961, 26, 1826-1831. doi:10.1021/jo01065a032

10. Garigipati, R. S. Tetrahedron Lett. 1990, 31, 1969-1972. doi:10.1016/S0040-4039(00)88891-7

11. Moriconi, E. J.; Cevasco, A. A. J. Org. Chem. 1968, 33, 2109-2111. doi:10.1021/jo01269a091

12. Vis, J. H.; Meinke, P. J. Heterocycl. Chem. 1970, 7, 1417-1419. doi:10.1002/jhet.5570070633

13. Yano, S.; Kazuno, H.; Sato, T.; Suzuki, N.; Emura, T.; Wierzba, K.; Yamashita, J.-i.; Tada, Y.; Yamada, Y.; Fukushima, M.; Asao, T. Bioorg. Med. Chem. 2004, 12, 3443-3450. doi:10.1016/j.bmc.2004.04.046

14. Kwok, R.; Pranc, R. J. Org. Chem. 1967, 32, 738-740. doi:10.1021/jo01278a052

15. Benati, L.; Bencivenni, G.; Leardini, R.; Nanni, D.; Minozzi, M.; Spagnolo, P.; Scialpi, R.; Zanardi, G. Org. Lett. 2006, 8, 2499-2502. doi:10.1021/ol0606637

16. Kato, T.; Takada, A.; Ueda, T. Chem. Pharm. Bull. 1972, 20, 901-904. doi:10.1248/cpb.20.901

17. Sharma, A.; Appukkuttan, P.; Van der Eycken, E. Chem. Commun. 2012, 48, 1623-1637. doi:10.1039/C1CC15238F

18. Yet, L. Chem. Rev. 2000, 100, 2963-3008. doi:10.1021/cr990407q

19. Molander, G. A. Acc. Chem. Res. 1998, 31, 603-609. doi:10.1021/ar960101v

20. Illuminati, G.; Mandolini, L. Acc. Chem. Res. 1981, 14, 95-102. doi:10.1021/ar00064a001

21. Galli, C.; Mandolini, L. Eur. J. Org. Chem. 2000, 3117-3125. doi:10.1002/1099-0690(200009)2000:18<3117::AID-EJOC3117>3.0.C 0;2-5

22. Michaut, A.; Rodriguez, J. Angew. Chem., Int. Ed. 2006, 45, 5740-5750. doi:10.1002/anie.200600787

23. López, S. E.; Restrepo, J.; Salazar, J. J. Chem. Res. 2007, 497-502. doi:10.3184/030823407X245598

24. Kanaoka, Y.; Kuga, T.; Tanizawa, K. Chem. Pharm. Bull. 1970, 18, 397-399. doi:10.1248/cpb.18.397

25. Kanaoka, Y.; Yoemitzu, O.; Sato, E.; Ban, Y. Chem. Pharm. Bull. 1968, 16, 280-284. doi:10.1248/cpb.16.280

26. Yildiz-Oren, I.; Yalcin, I.; Aki-Sener, E.; Ucarturk, N. Eur. J. Med. Chem. 2004, 39, 291-298. doi:10.1016/j.ejmech.2003.11.014

27. Kanaoka, Y.; Yonemitsu, O.; Tanizawa, K.; Ban, Y. Chem. Pharm. Bull. 1964, 12, 773-778. doi:10.1248/cpb.12.773

28. Kanaoka, Y.; Tanizawa, K.; Yoemitzu, O. Chem. Pharm. Bull. 1969, 17, 2381-2383. doi:10.1248/cpb.17.2381

29. Kanaoka, Y.; Sato, E.; Yonemitsu, O.; Ban, Y. Tetrahedron Lett. 1964, 5, 2419-2422. doi:10.1016/S0040-4039(00)76326-X

30. Loupy, A., Ed. Microwaves in Organic Synthesis; Wiley-VCH: Weinheim, 2002.

31. Kappe, C. O.; Dallinger, D. Nat. Rev. Drug Discovery 2006, 5, 51-63. doi:10.1038/nrd1926

32. Kappe, C. O. Angew. Chem., Int. Ed. 2004, 43, 6250-6284. doi:10.1002/anie.200400655

33. Shipe, W. D.; Wolkenberg, S. E.; Lindsley, C. W. Drug Discovery Today: Technol. 2005, 2, 155-161. doi:10.1016/j.ddtec.2005.05.002

34. Leadbeater, N. E. Chem. Commun. 2005, 2881-2902. doi:10.1039/b500952a 
35. Lidström, P.; Tierney, J.; Wathey, B.; Westman, J. Tetrahedron 2001, 57, 9225-9283. doi:10.1016/S0040-4020(01)00906-1

36. Perreux, L.; Loupy, A. Tetrahedron 2001, 57, 9199-9223. doi:10.1016/S0040-4020(01)00905-X

37. Caddick, S. Tetrahedron 1995, 51, 10403-10432. doi:10.1016/0040-4020(95)00662-R

38. Kappe, C. O.; Dallinger, D. Mol. Diversity 2009, 13, 71-193. doi:10.1007/s11030-009-9138-8

39. Van der Eicken, E.; Kappe, C. O. Microwave-Assisted Synthesis of Heterocycles; Distribution Center, $\mathrm{GmbH}, 2006$.

40. Bougrin, K.; Loupy, A.; Soufiaoui, M. J. Photochem. Photobiol., C: Photochem. Rev. 2005, 6, 139-167. doi:10.1016/j.jphotochemrev.2005.07.001

41. García, M. B.; Torres, R. A.; Orelli, L. R. Tetrahedron Lett. 2006, 47, 4857-4859. doi:10.1016/j.tetlet.2006.05.042

42. Díaz, J. E.; Bisceglia, J. Á.; Mollo, M. C.; Orelli, L. R. Tetrahedron Lett. 2011, 52, 1895-1897. doi:10.1016/j.tetlet.2011.02.042

43. Díaz, J. E.; Gruber, N.; Orelli, L. R. Tetrahedron Lett. 2011, 52, 6443-6445. doi:10.1016/j.tetlet.2011.09.097

44. Link, N. P.; Díaz, J. E.; Orelli, L. R. Synlett 2009, 751-754. doi:10.1055/s-0028-1087817

45. Yonemitsu, O.; Miyashita, K.; Ban, Y.; Kanaoka, Y. Tetrahedron 1969, 25, 95-100. doi:10.1016/S0040-4020(01)99460-8

46. Oklobdžija, M.; Šunjic, V.; Kajfež, F.; Čaplar, V.; Kolbah, D. Synthesis 1975, 596-598. doi:10.1055/s-1975-23847

\section{License and Terms}

This is an Open Access article under the terms of the Creative Commons Attribution License (http://creativecommons.org/licenses/by/4.0), which permits unrestricted use, distribution, and reproduction in any medium, provided the original work is properly cited.

The license is subject to the Beilstein Journal of Organic Chemistry terms and conditions:

(http://www.beilstein-journals.org/bjoc)

The definitive version of this article is the electronic one which can be found at: $\underline{\text { doi: } 10.3762 / \text { bjoc. } 12.190}$ 